\title{
Correlation and Path Analysis in Indian Mustard (Brassica juncea L.) for Seed Yield and Attributing Traits
}

\author{
Bhupendra Singh Yadav ${ }^{1 *}$, Hariom Kumar Sharma², \\ Ajay Pal Yadav ${ }^{1}$ and Bhagirath Ram $^{2}$ \\ ${ }^{1}$ MJRP University Jaipur Rajasthan, India \\ ${ }^{2}$ ICAR-Directorate of Rapeseed-Mustard Research, Bharatpur, Rajasthan, India \\ *Corresponding author
}

\section{A B S T R A C T}

\section{Keywords}

Correlation and path analysis, Mustard (Brassica juncea L.)

\section{Article Info}

\section{Accepted:}

15 January 2021

Available Online:

10 February 2021
An experiment was undertaken to find the correlation and path analysis of fourteen quantitative traits in 50 Indian mustard (Brassica juncea L.) germplasm lines. The experiment material was evaluated in a randomized block design with two replications. Analysis of variance ratios of the characters were found highly significant for the following traits seed yield per plant, fruiting zone length, first basal branch, days to initial flowering, days to $50 \%$ flowering. Coefficient of variation for GCV and PCV were found high for the following traits i.e. for First basal branch, Seed yield/plant (g), Thousand seed weight, Fruiting zone length, Seeds/siliqua. Higher heritability and higher genetic advance observed in the following characters seed yield per plant, thousand seed weight, fruiting zone length, days to initial flowering, first basal branch. Correlation study revealed that plant height, secondary branch, main shoot length, siliqua on main shoot, maturity exerted high positive significant genotypic correlation with seed yield per plant. In phenotypic correlation was observed that main shoot length, siliqua on main shoot, maturity showed highly significant and positive correlation with seed yield per plant. Path analysis revealed that seeds per siliqua showed maximum positive direct genotypic effect however the highest direct phenotypic effect of initial flowering was exhibited through seed yield per plant.

\section{Introduction}

Indian Mustard (Brassica juncea L. czern.\&coss.), common name-Indian mustard, Family- Brassicaceae or Cruciferae and Chromosome number $(2 \mathrm{n}=36)$. Botanically, Indian mustard [Brassica juncea (L.) Czern.\& Coss.] $(2 \mathrm{n}=36$, AABB genome), is an allopolyploid commonly called as Indian mustard, contributes more than $80 \%$ to the total rapeseed-mustard production in the country and is an important component in the oilseed sector. It is mainly self-pollinating, although an average of 7.5 to 30 percent outcrossing does occur under natural field conditions. Indian mustard (Brassica juncea 
(L.) is an important Rabi season crop extensively grown as under irrigated condition. Yield is complex character which dependent on the various yield contributing traits.

Thus, the study of correlation between yield and its component is of primary importance in formulating the selection criteria under crop improvement. it is necessary to know the various components of the yield and its mutual correlation with other independent traits. This is because, selection would be more efficient if it is based on some components which less sensitive to environment. It is well known that correlation mainly does not fulfill the purpose of the researcher because it does not detect the characters having indirect effects on seed yield.

In such situation path coefficient analysis developed by Wright (1921) put forward the real importance of such characters of partitioning the correlation coefficient in to direct as well as indirect effects.

India is the largest agrarian subcontinent supporting $26 \%$ world's agricultural population on $12 \%$ arable land. India is also the fifth largest vegetable oil economy accounting 7.4\%oilseeds, 5.8\%oils and 6.1\% oil meal production, and $9.3 \%$ of edible oil consumption in the world. Oilseeds are the second most important agricultural economy in India next to cereals growing at a pace of $4.1 \%$ per annum in the last three decades. Oilseed brassica shares $23.5 \%$ area and $24.2 \%$ production of total oilseeds in the country.

Despite being the third largest producer $(11.3 \%)$ of oilseed brassica after Canada and China in the world, India meets $57 \%$ of the domestic edible oil requirements through imports and ranked 7th largest importer of edible oils in the world. (Jat et al., 2019).

\section{Materials and Methods}

The materials for the present investigation consisted of 50 genotypes of Indian mustard obtained from germplasm unit of ICARDirectorate of Rapeseed-Mustard Research, Sewar, Bharatpur. 50 genotypes of Indian mustard including 5 check varieties (NPJ-112, Pusa bold, PM-26, CS-56, Kranti) were evaluated in RCBD with two replications under irrigated timely sown condition.

Sowing was done on 15-10-2019. In each replication each genotype was sown in a plot of one row of 3-meter length. Plant to plant spacing were maintained at $10 \mathrm{~cm}$ by thinning after 15-20 days of sowing. Observation Recorded five competitive plants were randomly selected at the time of maturity (except the days to initial flowering, days to 50 per cent flowering and days to maturity which were recorded on plot basis) Plant height $(\mathrm{cm})$, Primary branches per plant, Secondary branches per plant, Main shoot length $(\mathrm{cm})$, Siliqua on main shoot, Fruiting zone length $(\mathrm{cm})$, First basal branching $(\mathrm{cm})$, Initial flowering, Days to 50 percent flowering, Days to maturity, Siliqua length $(\mathrm{cm})$, Seeds per siliqua, Seed yield per plant (g), Thousand seed weight $(\mathrm{g})$.

These traits were computed on basis of mean data after computing for each character was subjected to standard method of analysis of variance following Panse and Sukhatme (1978) phenotypic and genotypic coefficient of variation, heritability (Broad Sense) and genetic advance as percent of mean were estimated by the formula al suggested by Burton (1952) and Johnson et al., (1955). The genotypic correlation coefficients were estimated according to the formula given by Al-Jibouri et al., (1958) while path analysis was carried out using the genotypic correlation coefficient to know direct and indirect effects of the components on yield as suggested by 
Wright (1921) and illustrated by Dewey and Lu (1957).

\section{Results and Discussion}

Correlation studies were also great interest for plant breeders in determining the traits which are correlated with main breeding objectives. The genotypic and phenotypic correlations between the fourteen characters are also summarized (Table 1 and 2) at 1\% and 5\% significant level. A positive and highly significant genotypic character association was found between Plant height and fruiting zone length $\left(1.093^{* *}\right)$, first basal branch $(0.596 * *)$, initial flowering $(0.591 * *), 50 \%$ flowering $\left(0.436^{* *}\right)$, seed yield per plant $\left(0.275^{* *}\right)$, thousand seed weight $\left(0.278^{* *}\right)$ and showed significant and positive correlation with siliqua length $(0.199 *)$. Main shoot length had highly significant and positive association with siliqua on main shoot $(0.701 * *)$, initial flowering $(0.299 *)$, maturity $(0.372 * *)$, seed yield per plant $(0.564 * *)$, seed per siliqua $(0.522 * *)$, thousand seed weight $(0.359 * *)$. Seed yield per plant showed highly significant and positive association with seed per siliqua $(0.398 * *)$. A positive and highly significant genotypic character association between silique per plant and yield per plant was also found by Tuncturk and Ciftci (2007).Highly positive correlation found between plant height and yield per plant, silique per plant and yield per plant by Khayat et al., (2012). A positive and highly significant phenotypic correlation was found between Plant height and main shoot length $\left(0.198^{*}\right)$, siliqua on main shoot $\left(0.209^{*}\right)$, fruiting zone length $(0.624 * *)$, first basal branch $(0.277 * *)$, initial flowering $(0.282 * *)$. Main shoot length showed highly significant and positive association with siliqua on main shoot $\left(0.338^{* *}\right)$, seed yield per plant $\left(0.269^{* *}\right)$ and significant with thousand seed weight $\left(0.216^{*}\right)$. Seed yield per plant showed significant and positive association with seed per siliqua $\left(0.226^{*}\right)$, thousand seed weight $\left(0.211^{*}\right)$. The correlation studies revealed that the most important traits in selection for yield are plant height, days to maturity, seeds per plant and silique per plant. Moreover, in analysis most of the correlated pair of characters, genotypic and phenotypic association were in the same direction. The genotypic estimates were higher than phenotypic estimates indicating that an inherited association occurred between traits. Almost similar results are given by Rameeh (2011), while these results are also partial agreement with the earlier findings of Dar et al., (2010); Tahira et al., (2012). Path coefficient analysis splits the correlation coefficient into direct and indirect genotypic effects. It reveals whether the association of the traits with yield is due to their direct effect or is a consequence of their indirect effect via other traits.

The data (Table 3) revealed that highest positive direct effect on seeds per siliqua (3.126) and this character was followed by secondary branches (2.694), siliqua on main shoot (1.776), fruiting zone length (1.167), siliqua length (0.988), maturity (0.863), primary branches (0.579), plant height (0.564), initial flowering (0.548) and thousand seed weight $(0.499)$ was positive on seed yield per plant. Path coefficient analysis splits the correlation coefficient into direct and indirect phenotypic effects. It reveals whether the association of the traits with yield is due to their direct effect or is a consequence of their indirect effect via other traits. The data (Table 4) revealed that highest positive direct effect on initial flowering (0.329) and this character was followed by seeds per siliqua (0.272), siliqua on main shoot (0.250), maturity (0.175), plant height (0.156), thousand seed weight (0.148), secondary branches (0.111) and main shoot length (0.068), was positive on seed yield per plant. 
Table.1 Genotypic correlations between different quantitative traits of Indian mustard

\begin{tabular}{|c|c|c|c|c|c|c|c|c|c|c|c|c|c|}
\hline & $\begin{array}{c}\text { Plant } \\
\text { height }\end{array}$ & $\begin{array}{l}\text { Primary } \\
\text { branches }\end{array}$ & $\begin{array}{c}\text { Secondary } \\
\text { branches }\end{array}$ & $\begin{array}{l}\text { Main } \\
\text { shoot } \\
\text { length }\end{array}$ & $\begin{array}{c}\text { Siliqua on } \\
\text { main } \\
\text { shoot }\end{array}$ & $\begin{array}{l}\text { Fruiting } \\
\text { zone } \\
\text { length }\end{array}$ & $\begin{array}{c}\text { First basal } \\
\text { branch }\end{array}$ & $\begin{array}{c}\text { Days to } \\
\text { initial } \\
\text { flowering }\end{array}$ & $\begin{array}{c}\text { Days } \\
50 \% \\
\text { flowering }\end{array}$ & $\begin{array}{l}\text { Days to } \\
\text { maturity }\end{array}$ & $\begin{array}{c}\text { Seed yield/ } \\
\text { plant }\end{array}$ & $\begin{array}{l}\text { Siliqua } \\
\text { length }\end{array}$ & $\begin{array}{l}\text { Seeds/ } \\
\text { siliqua }\end{array}$ \\
\hline $\begin{array}{c}\text { Primary } \\
\text { branch }\end{array}$ & 0.073 & & & & & & & & & & & & \\
\hline $\begin{array}{c}\text { Secondary } \\
\text { branch }\end{array}$ & $-0.247^{*}$ & $0.713^{* *}$ & & & & & & & & & & & \\
\hline $\begin{array}{l}\text { Main shoot } \\
\text { length }\end{array}$ & -0.076 & $0.585^{* *}$ & $0.588^{* *}$ & & & & & & & & & & \\
\hline $\begin{array}{l}\text { Siliqua on } \\
\text { main shoot }\end{array}$ & $-0.28^{* *}$ & $0.513^{* *}$ & $0.525^{* *}$ & $0.701^{* *}$ & & & & & & & & & \\
\hline $\begin{array}{c}\text { Fruiting } \\
\text { zone length }\end{array}$ & $1.093^{\text {** }}$ & 0.097 & -0.116 & 0.047 & $-0.207^{*}$ & & & & & & & & \\
\hline $\begin{array}{c}\text { First basal } \\
\text { branch }\end{array}$ & $0.596^{* *}$ & -0.013 & $-0.389^{* *}$ & -0.074 & $-0.264^{* *}$ & $0.537^{* *}$ & & & & & & & \\
\hline $\begin{array}{c}\text { Days Initial } \\
\text { flowering }\end{array}$ & $0.591^{* *}$ & $0.636^{* *}$ & $0.438^{* *}$ & $0.299^{* *}$ & 0.070 & $0.528^{* *}$ & $0.450^{* *}$ & & & & & & \\
\hline $\begin{array}{l}\text { Days to } 50 \\
\% \text { flowering }\end{array}$ & $0.436^{* * *}$ & $0.690^{* *}$ & $0.406^{* *}$ & 0.174 & $-0.226^{*}$ & $0.439^{* *}$ & $0.555^{* *}$ & $0.903^{* *}$ & & & & & \\
\hline $\begin{array}{l}\text { Days to } \\
\text { maturity }\end{array}$ & -0.056 & $0.529^{* *}$ & $0.209^{*}$ & $0.372^{* *}$ & -0.028 & $-0.352^{* *}$ & 0.181 & $-0.331^{* *}$ & $-0.708^{* *}$ & & & & \\
\hline $\begin{array}{l}\text { Seed yield/ } \\
\text { plant (g) }\end{array}$ & $0.275^{* *}$ & 0.019 & $0.264^{* *}$ & $0.564^{* *}$ & $0.804^{* *}$ & 0.075 & 0.059 & -0.014 & $-0.211^{*}$ & $0.216^{*}$ & & & \\
\hline $\begin{array}{l}\text { Siliqua } \\
\text { length }\end{array}$ & $0.199^{*}$ & $0.214^{*}$ & 0.166 & 0.171 & -0.192 & 0.145 & $0.321^{* *}$ & $0.264^{* *}$ & $0.318^{* *}$ & $-0.361^{* *}$ & -0.031 & & \\
\hline $\begin{array}{l}\text { Seeds/ } \\
\text { siliqua }\end{array}$ & 0.026 & $-0.229^{*}$ & -0.145 & $0.522^{* *}$ & $0.240^{*}$ & 0.106 & -0.056 & -0.085 & -0.023 & -0.109 & $0.398^{* *}$ & -0.124 & \\
\hline $\begin{array}{l}\text { Thousand } \\
\text { seed weight }\end{array}$ & $0.278^{* *}$ & -0.068 & -0.107 & $0.359^{* *}$ & $0.258^{* *}$ & $0.224^{*}$ & 0.180 & -0.076 & -0.084 & -0.150 & 0.186 & 0.176 & $0.265^{* *}$ \\
\hline
\end{tabular}

$*$ and ** Significance at $5 \%$ and $1 \%$ level of significance, respectively. 
Table.2 Phenotypic correlations between different quantitative traits of Indian mustard

\begin{tabular}{|c|c|c|c|c|c|c|c|c|c|c|c|c|c|}
\hline & $\begin{array}{c}\text { Plant } \\
\text { height }\end{array}$ & $\begin{array}{l}\text { Primary } \\
\text { branches }\end{array}$ & $\begin{array}{c}\text { Secondary } \\
\text { branches }\end{array}$ & $\begin{array}{l}\text { Main } \\
\text { shoot } \\
\text { length }\end{array}$ & $\begin{array}{c}\text { Siliqua } \\
\text { on } \\
\text { main } \\
\text { shoot }\end{array}$ & $\begin{array}{c}\text { Fruiting } \\
\text { zone } \\
\text { length }\end{array}$ & $\begin{array}{c}\text { First } \\
\text { basal } \\
\text { branch }\end{array}$ & $\begin{array}{c}\text { Days to } \\
\text { initial } \\
\text { flowering }\end{array}$ & $\begin{array}{c}\text { Days } \\
\mathbf{5 0} \% \\
\text { flowering }\end{array}$ & $\begin{array}{l}\text { Days to } \\
\text { maturity }\end{array}$ & $\begin{array}{c}\text { Seed } \\
\text { yield/ } \\
\text { plant }\end{array}$ & $\begin{array}{l}\text { Siliqua } \\
\text { length }\end{array}$ & $\begin{array}{l}\text { Seeds/ } \\
\text { siliqua }\end{array}$ \\
\hline $\begin{array}{l}\text { Primary } \\
\text { branch }\end{array}$ & 0.145 & & & & & & & & & & & & \\
\hline $\begin{array}{l}\text { Secondary } \\
\text { branch }\end{array}$ & 0.125 & $0.705^{* * *}$ & & & & & & & & & & & \\
\hline $\begin{array}{l}\text { Main shoot } \\
\text { length }\end{array}$ & $0.198^{*}$ & 0.196 & 0.121 & & & & & & & & & & \\
\hline $\begin{array}{l}\text { Siliqua on } \\
\text { main shoot }\end{array}$ & $0.209^{*}$ & $0.268^{* * *}$ & $0.403^{* *}$ & $0.338^{* * *}$ & & & & & & & & & \\
\hline $\begin{array}{c}\text { Fruiting } \\
\text { zone length }\end{array}$ & $0.624^{* * *}$ & -0.069 & -0.082 & 0.152 & 0.051 & & & & & & & & \\
\hline $\begin{array}{c}\text { First basal } \\
\text { branch }\end{array}$ & $0.277^{* *}$ & -0.182 & $-0.338^{* *}$ & 0.070 & -0.014 & $0.386^{* *}$ & & & & & & & \\
\hline $\begin{array}{c}\text { Days Initial } \\
\text { flowering }\end{array}$ & $0.282^{* *}$ & $0.197^{*}$ & 0.180 & 0.144 & 0.022 & $0.349^{* * *}$ & 0.050 & & & & & & \\
\hline $\begin{array}{c}\text { Days to } 50 \\
\% \\
\text { flowering }\end{array}$ & 0.183 & 0.163 & $0.205^{*}$ & 0.036 & -0.038 & $0.206^{*}$ & -0.031 & $0.835^{* *}$ & & & & & \\
\hline $\begin{array}{l}\text { Days to } \\
\text { maturity }\end{array}$ & 0.030 & 0.134 & $0.256^{*}$ & -0.122 & 0.136 & -0.020 & -0.117 & -0.042 & -0.046 & & & & \\
\hline $\begin{array}{l}\text { Seed yield/ } \\
\text { plant (g) }\end{array}$ & 0.148 & 0.087 & 0.186 & $0.269^{* * *}$ & $0.366^{* *}$ & 0.042 & -0.026 & 0.051 & -0.037 & $0.201^{*}$ & & & \\
\hline $\begin{array}{l}\text { Siliqua } \\
\text { length }\end{array}$ & 0.135 & -0.008 & 0.053 & -0.019 & -0.076 & 0.170 & 0.160 & 0.058 & 0.091 & -0.140 & -0.123 & & \\
\hline $\begin{array}{l}\text { Seeds/ } \\
\text { siliqua }\end{array}$ & 0.069 & 0.074 & -0.007 & 0.155 & 0.061 & 0.117 & -0.062 & -0.075 & -0.091 & -0.037 & $0.226^{*}$ & 0.147 & \\
\hline $\begin{array}{c}\text { Thousand } \\
\text { seed weight }\end{array}$ & 0.091 & 0.011 & -0.026 & $0.216^{*}$ & 0.116 & 0.147 & 0.125 & -0.174 & -0.112 & -0.118 & $0.211^{*}$ & 0.068 & 0.157 \\
\hline
\end{tabular}

$*$ and $* *$ Significance at $5 \%$ and $1 \%$ level of significance, respectively. 
Table.3 Direct and indirect effect of yield components on seed yield (Genotypic)

\begin{tabular}{|c|c|c|c|c|c|c|c|c|c|c|c|c|c|c|}
\hline & PHT & PB & SB & MSL & SMS & FZL & FBB & IF & $\mathbf{5 0 F}$ & MAT & SL & SPS & TSW & r2 with \\
SYP
\end{tabular}

Residual are -0.46618 
Table.4 Direct and indirect effect of yield components on seed yield (Phenotypic)

\begin{tabular}{|c|c|c|c|c|c|c|c|c|c|c|c|c|c|c|}
\hline & PHT & PB & SB & MSL & SMS & FZL & FBB & IF & $\mathbf{5 0 F}$ & MAT & SL & SPS & TSW & r2 with \\
SYP
\end{tabular}

Residual are 0.69449 
Similar result also reported by Singh and Singh (2010), Mahla et al., (2003), and Kumar et al., (2016). Seed per plant was the variable with maximum potential of selection for seed yield improvement because this trait possessed high h2 B.S, highly significant positive correlation and maximum positive direct effects with yield.

\section{References}

Al-Jibouri H.A., Miller P.A., Robinson H.F. 1958. Genotype and environmental variances and co-variance in upland cotton cross of interspecific origin. Agron. J. 50:633-637.

Burton G.W. 1952. Quantitative Inheritance in Grasses in. Proc. 6th Int. Grassland Congress. 7:273-283.

Dar Z.A., Wani S.A., Zaffar G., Ishfaq A., Wani M.A., Habib M., Khan M.H., Razvi S.M., 2010. Character association and path coefficient studies in Brown sarson (Brassica rapa L.). Res. J. Agri. Sci.1(2):153-154.

Dewey D.R., Lu K.H. 1957. A correlation and path coefficient analysis of components of crested wheat grass seed production. Agron. J. 5(1):515-518.

Jat R.S., Singh V.V., Sharma P., Rai P.K. 2019. Oilseed brassica in India: Demand, supply, policy perspective and future potential. OCL 26:8.

Johnson H.W., Robinson H.F., Comstock R.E. 1955. Estimates of genetic and environmental variability in soybean. Agron. J. 47:314-318.

Khayat M., Lack Sh., KaramiH., 2012. Correlation and path analysis of traits affecting grain yield of canola (Brassica napus L.) varieties. J. Basic. Appl. Sci. Res. 2(6): 5555-5562.

Panse V.G., Sukhatme P.V. 1978.Statistical methods for Agricultural Workers. ICAR, New Delhi. 235-246.

Rameeh V. 2011. Correlation and path analysis in advanced lines of rapeseed (Brassica napus) for yield components. J. Oilseed Brassica. 2(2):56-60.

Tahira T., Mahmood M.S., Tahir U., Saleem M., Hussain M., Saqib. 2012. The estimation of heritability, association and selection criteria for yield components in mustard (Brassica juncea). Pak. J. Agri. Sci. 48(4):251-254.

Tuncturk M., CiftciV. 2007. Relationships between yield and some yield components in rapeseed (Brassica napus ssp. oleifera L.) cultivars by using correlation and path analysis. Pak. J. Bot. 39(1):81-84.

Wright S. 1921. Correlation and causation. J. Agric. Res. 1(20): 557-585.

Singh S.K., Singh A.K. 2010. Inter-relationship and path analysis for seed yield in Indian mustard. Indian J Ecol. 3(7):8-12.

Mahla H.R., Jambhulkar S.J., Yadav D.K., Sharma R. 2003. Genetic variability, correlation and path analysis in Indian mustard [Brassica juncea (L.) Czern. and Coss.]. Indian $J$ Genet Plant Breed. 6(3):171-172.

Kumar Rajeev., Gaurav S.S., Jayasudha S., Kumar Hitesh. Study of correlation and path coefficient analysis in germplasm lines of Indian mustard (Brassica juncea L.). Agricultural Science Digest. 36(2):9296.

\section{How to cite this article:}

Bhupendra Singh Yadav, Hariom Kumar Sharma, Ajay Pal Yadav and Bhagirath Ram. 2021. Correlation and Path Analysis in Indian Mustard (Brassica juncea L.) for Seed Yield and Attributing Traits. Int.J.Curr.Microbiol.App.Sci. 10(02): 1761-1768.

doi: https://doi.org/10.20546/ijcmas.2021.1002.208 